\title{
Hemostatic profile under fluid resuscitation during rivaroxaban anticoagulation: an in vitro survey
}

Helin, T. A.

2018-12

Helin , T A , Zuurveld , M , Manninen , M , Meijers , J C M , Lassila , R \& Brinkman , H J M 2018 , ' Hemostatic profile under fluid resuscitation during rivaroxaban anticoagulation: an in vitro survey ' , Transfusion , vol. 58 , no. 12 , pp. 3014-3026 . https://doi.org/10.1111/trf.14933

http://hdl.handle.net/10138/285686

https://doi.org/10.1111/trf.14933

submittedVersion

Downloaded from Helda, University of Helsinki institutional repository.

This is an electronic reprint of the original article.

This reprint may differ from the original in pagination and typographic detail.

Please cite the original version. 


\section{Hemostatic profile under fluid resuscitation during rivaroxaban anticoagulation}

\section{- an in vitro survey}

Tuukka A Helin ${ }^{1}$, Marleen Zuurveld², Mikko Manninen ${ }^{3}$, Joost CM Meijers ${ }^{2,4}$, Riitta Lassila ${ }^{1}$, Herm Jan M Brinkman²\#

${ }^{1}$ Coagulation Disorders Unit, Clinical Chemistry, HUSLAB Laboratory Services, Helsinki University Hospital, Helsinki, Finland

${ }^{2}$ Department of Plasma Proteins, Sanquin Research, Amsterdam, The Netherlands

${ }^{3}$ Orton Orthopaedic Hospital, Helsinki, Finland

${ }^{4}$ Department of Experimental Vascular Medicine. Academic Medical Center, University of Amsterdam, Amsterdam, The Netherlands

\# To whom correspondence should be addressed

Address for correspondence:

Dr HJM Brinkman, PhD

Sanquin Research

Dep. Plasma Proteins

Plesmanlaan 125

1066 CX Amsterdam

The Netherlands

+3120 5121289

Reprints will not be available from the author

Sources of support: None

Disclosure of Conflict of Interest

MZ, JCMM and HJMB are employed by Sanquin, manufacturer of PCC and distributor of S/D-treated plasma in the Netherlands. RL is an advisory board member of Bayer in Finland and of LFB in Europe. TH and MM declare no conflicts of interest.

This is the pre-peer reviewed version of the following article: Helin TA, Zuurveld M, Manninen M, Meijers JCM, Lassila R, Brinkman HJM. Hemostatic profile under fluid resuscitation during rivaroxaban anticoagulation: an in vitro survey. Transfusion. 2018 58:3014-26. doi:

10.1111/trf.14933, which has been published in final form at https://onlinelibrary.wiley.com/doi/full/10.1111/trf.14933. This article may be used for noncommercial purposes in accordance with Wiley Terms and Conditions for Use of Self-Archived Versions. 


\section{Abstract}

\section{BACKGROUND}

Uncontrollable bleeding is the leading cause of death in traumatically injured patients. The extent to which direct factor Xa inhibitors (FXa-I) interfere with the applied resuscitation measures is presently unknown.

\section{STUDY DESIGN AND METHODS}

In this study we investigated the effect of the resuscitation fluids saline, albumin, fresh frozen plasma (FFP) and solvent/detergent (S/D)-treated plasma, fibrinogen concentrate, prothrombin complex concentrate (PCC) and combinations thereof on the hemostatic profile of rivaroxaban-anticoagulated whole blood and plasma.

We used rivaroxaban-spiked whole blood and plasma from healthy donors, as well as plasma from patients on rivaroxaban and mimicked a resuscitation approach in a $50 \%$ plasma dilution setting. Thromboelastography (TEG), thrombin generation (CAT) and fibrin generation clot lysis test (FGCLT) were assessed using tissue factor (TF) to initiate coagulation and tissue plasminogen activator (tPA) to induce clot lysis.

\section{RESULTS}

Rivaroxaban resulted in a hypo-coagulant state that remained largely unaltered upon subsequent $50 \%$ dilution with S/D-treated plasma or FFP. Using S/D-treated plasma as a diluent, clot stability decreased due to its low $\alpha_{2}$-antiplasmin. Dilution with saline and albumin induced a profibrinolytic state and further deteriorated the impaired hemostatic potential of rivaroxaban-anticoagulated blood, even after PCC and fibrinogen support. Combined use of plasma (either FFP or S/D-treated) and PCC, however, considerably improved both coagulation and clot stability.

\section{CONCLUSION}


In the setting of rivaroxaban anticoagulation and major bleed and trauma, transfusing plasma together with PCC may provide the most effective resuscitation approach with the notion that additional antifibrinolytic drug support (e.g. tranexamic acid) might be required.

Key Words: rivaroxaban, fluid resuscitation, solvent/detergent plasma, fresh frozen plasma, prothrombin complex concentrate, thromboelastography, thrombin generation, calibrated automated thrombogram, fibrin generation clot lysis test 


\section{INTRODUCTION}

Uncontrollable hemorrhage is the leading cause of death in traumatically injured patients. ${ }^{1}$ Hampered coagulation together with hyperfibrinolysis is a condition that may play a major role in this lifethreatening coagulopathy. ${ }^{2-4}$ In an attempt to secure intravascular volume and tissue oxygenation, crystalloid fluids and red blood cells are frequently transfused as the first-line treatment of severe hemorrhagic shock. ${ }^{5,6} \mathrm{~A}$ strategy to avoid coagulopathy includes the administration of fibrinogen concentrate, anti-fibrinolytic drug tranexamic acid (TXA), platelets and plasma. ${ }^{7,8}$ For resuscitation with plasma, single donor fresh frozen plasma (FFP) or solvent/detergent (S/D)-treated plasma is used, the latter consisting of a blood group specific pool of over 600 single donor units (treated with tri-n-butyl phosphate and $1 \%$ Triton X-100). Major advantages of S/D-treated plasma over FFP are the betweenbatch homogeneity, the reduced risk of pathogen transmission and its safety with respect to transfusion-related acute lung injury and lower likelihood of allergic reactions. ${ }^{9}$ Disadvantage of S/Dtreated plasma may be the low level of $\alpha 2$-antiplasmin and the concomitant hyperfibrinolytic phenotype, which on the other hand may be counteracted by its enhanced coagulation activity due to loss of protein S function. ${ }^{10}$ Prothrombin complex concentrate (PCC), a mixture of partly purified human plasma vitamin K-dependent coagulation factors (F) (FII, VII, IX, X, protein C and S) can be considered in combination with or as an alternative to plasma. ${ }^{11-13}$

The direct factor Xa inhibitor rivaroxaban, among others, is used for stroke prevention with atrial fibrillation, as well as thromboprophylaxis in patients undergoing orthopedic surgery and treatment of venous thromboembolism (VTE). ${ }^{14-16}$ For bleeding management in patients taking rivaroxaban, firstly, drug intake is discontinued. In cases of severe bleeds, e.g. during trauma, drug cessation is not sufficient and reversal agents must be used. ${ }^{17} \mathrm{~A}$ specific reversal agent, andexanet alfa, is still in phase III trials, ${ }^{18}$ and not yet available. PCC has not only shown rapid normalization of the laboratory variables prothrombin time (PT) and endogenous thrombin potential (ETP) in healthy volunteers taking rivaroxaban, ${ }^{19,20}$ but PCC also corrected hemorrhage in animal bleeding models. ${ }^{21-23}$ 
The use of rivaroxaban or other factor Xa inhibitors may add to the development of trauma- or surgery-induced coagulopathy. Simultaneous fluid replacement with albumin, salt solutions or plasma may not only affect the coagulation profile due to a lower hematocrit, platelet count and dilution of coagulation and fibrinolytic proteins (especially with $\mathrm{NaCl}$ and albumin), but also lowers rivaroxaban levels in plasma.

Herein, we employed an in vitro coagulopathy model with combined hyperfibrinolysis and rivaroxaban anticoagulation and studied different resuscitation approaches, including the use of $0.9 \% \mathrm{NaCl}$, fibrinogen, PCC, S/D-treated plasma, tranexamic acid and their combinations. We also tested the effects of these regimes in diluted plasma from orthopedic patients taking their first dose of rivaroxaban $16-28 \mathrm{~h}$ after the surgery. 


\section{MATERIALS AND METHODS}

\section{Blood collection and plasma preparation}

Blood samples were collected from apparently healthy volunteer donors after informed consent by standard venipuncture using a 21-gauge needle and $10 \mathrm{~mL}$ vacuum collection tubes containing trisodium citrate (109 mM). For TEG analysis, samples were kept at room temperature and used within $5 \mathrm{~h}$ after blood collection. Plasma was prepared by centrifugation of citrated whole blood for 10 min at $2000 \mathrm{~g}$ within $2 \mathrm{~h}$ after venipuncture. Platelet-poor plasma (PPP) was collected, aliquoted, snapfrozen in liquid nitrogen and stored at $-20^{\circ} \mathrm{C}$ or below until further use. In vitro anticoagulation was achieved with rivaroxaban (Bayer Healthcare, Leverkusen, Germany) at a plasma concentration of 50 $\mathrm{ng} / \mathrm{mL}$ (trough value) and $300 \mathrm{ng} / \mathrm{mL}$ (peak value) by spiking $3 \mu \mathrm{L}$ rivaroxaban dilutions in DMSO to 1 $\mathrm{mL}$ plasma (for whole blood assuming a hematocrit of $40 \%$ ). Non-anticoagulated samples contained an equal amount of the DMSO solvent. Alternatively, plasma was prepared from citrated whole blood collected from orthopedic patients who underwent total hip arthroplasty and taking their first dose of rivaroxaban. Written informed consent was obtained prior to blood withdrawal. The sample was taken at 3 hours after the first rivaroxaban oral dose to obtain peak levels, at 16-28 hours after the surgery. PPP was prepared, frozen and stored until analysis. Rivaroxaban concentration was analyzed with a rivaroxaban-calibrated anti-Xa assay (Instrumentation Laboratory Rivaroxaban calibrators) with an Instrumentation Laboratory ACL Top 500 analyser.

\section{Resuscitation fluids, coagulation factor concentrates and inhibitors}

Normal pooled plasma was prepared from 32 FFP units (Sanquin, Amsterdam, The Netherlands). S/D-treated plasma (blood group A) was from Octapharma AG (Lachen, Switzerland). PCC (Cofact) and $5 \%$ albumin solution (Albuman) were from Sanquin. Saline $(0.9 \% \mathrm{NaCl})$ was from Fresenius Kabi (Bad Homburg, Germany). Fibrinogen (Haemocomplettan P) and tranexamic acid (laboratory grade) were purchased from CLS Behring (King of Prussia, PA, USA) and Sigma-Aldrich (Darmstadt, Germany), respectively. Potato carboxypeptidase inhibitor was from Sigma Aldrich. $\alpha_{2}$-antiplasmin was obtained from Mybiosource (Vancouver, Canada). 


\section{In vitro coagulation models}

We employed two model systems for hyperfibrinolysis: viscoelastic measurement in whole blood (TEG) and a cell-free fibrin generation clot lysis test (FGCLT) in PPP with optical detection of fibrin formation and clot lysis. In both models, coagulation was triggered by tissue factor (TF) and fibrinolysis by tissue plasminogen activator (tPA). Thrombin generation assay (calibrated automated thrombography, CAT) in the absence of tPA was employed as a dedicated test solely to investigate the effect of resuscitation on the anticoagulant effect of rivaroxaban.

Whole blood thromboelastography (TEG: Haemonetics, MA, USA) was performed with minimally altered citrate-anticoagulated whole blood (containing rivaroxaban or DMSO) and samples thereof diluted with plasma, saline or albumin solution. To avoid unwanted red cell agglutination during TEG analysis upon whole blood dilution with pooled plasma, blood from blood group $\mathrm{O}$ donors was used for experimentation only. The added volume of resuscitation fluid was equal to the volume of plasma in the original whole blood sample, assuming a hematocrit of $40 \%$ (the range of hematocrit in the citrated whole blood samples was 36 - 45\%). Other reagents and supplements were added just before the start of the TEG measurements, being TF (Innovin: Siemens Healthcare Diagnostics), tPA (Actilyse: Boehringer Ingelheim Gmbh) and when indicated tranexamic acid, fibrinogen, $\alpha_{2}$-antiplasmin and PCC. All reagents and supplements were diluted in Tris-buffered saline $(10 \mathrm{mM}$ Tris, $150 \mathrm{mM} \mathrm{NaCl}$, $\mathrm{pH}$ 7.4) and their addition inevitably further diluted the blood sample. We confined this extra dilution to a minimum and confirmed in all samples that the volume of added reagents and supplements accounted for $14 \% \mathrm{v} / \mathrm{v}$ of the total extracellular volume. Final concentration of TF, tPA and tranexamic acid were respectively $1 \mathrm{pM}, 200 \mathrm{ng} / \mathrm{mL}$ and $2.5 \mu \mathrm{g} / \mathrm{mL}$. PCC was added to equal a concentration in undiluted plasma of $2 \mathrm{IU} / \mathrm{mL}$. Fibrinogen and $\alpha_{2}$-antiplasmin $(2 \mathrm{mg} / \mathrm{mL}$ and $1 \mathrm{U} / \mathrm{mL}$, respectively) were added to the saline compartment to restore their normal levels. S/D-treated plasma was supplemented with $0.6 \mathrm{IU} / \mathrm{mL} \alpha 2$-antiplasmin to restore its value to $1 \mathrm{IU} / \mathrm{mL}$. Finally, $340 \mu \mathrm{L}$ prepared sample was pipetted into a TEG cup and measurement was started after adding $20 \mu \mathrm{L} 0.2 \mathrm{M} \mathrm{CaCl}_{2}$. 
The cell-free FGCLT was performed as described by Bakhtiari et al. ${ }^{24}$ In this assay, the final plasma concentration was $50 \% \mathrm{v} / \mathrm{v}$. The remaining $50 \% \mathrm{v} / \mathrm{v}$ was Tris-buffered saline, containing additives (PCC or carboxypeptidase inhibitor when indicated) and start reagents (TF, phospholipids, tPA and $\mathrm{CaCl}_{2}$ at final concentrations of respectively $0.5 \mathrm{pM}, 4 \mu \mathrm{M}, 50 \mathrm{ng} / \mathrm{mL}$ and $15 \mathrm{mM}$ ). This assay measures turbidity $(405 \mathrm{~nm})$ of clotting plasma in time. The first part of the optical density (OD) tracing from zero to maximum turbidity characterizes clot formation, with clotting time (CT) defined as the time from start of the measurement to half maximal turbidity (midpoint of the clear to maximum turbid transition). The second part of the tracing with declining OD represents clot lysis. Clot lysis time (CLT) was defined as the time between $\mathrm{CT}$ and half maximal lysis (midpoint of the maximum turbid to clear transition). Plasma samples were subjected to FGCLT either directly or after dilution with an equal amount of pooled FFP or S/D-treated plasma.

CAT reagents were obtained from Thrombinoscope-Stago (Maastricht, The Netherlands). CAT was performed with donor plasma directly and after $50 \%$ dilution with either S/D-treated plasma or pooled FFP. As per manufacturer's instruction, the CAT assay should be performed at a $66.7 \% \mathrm{v} / \mathrm{v}$ final plasma concentration ( $80 \mu \mathrm{L}$ plasma, $20 \mu \mathrm{L}$ reagents containing TF and phospholipids or $20 \mu \mathrm{L}$ calibrator, $20 \mu \mathrm{L}$ start reagents containing $\mathrm{CaCl}_{2}$ and the thrombin substrate). To be able to add supplements like PCC, we lowered the final plasma concentration to 50\%, compatible with our FGCLT and as reported previously. ${ }^{25}$ TF concentration was 1 or $5 \mathrm{pM}$.

\section{Statistical analysis}

The GraphPad Prism 7 package (GraphPad Software, Inc) was used for statistical calculations.

Differences were considered significant at $\mathrm{p}<0.05$. 


\section{RESULTS}

\section{Reduction of rivaroxaban concentration by plasma dilution; influence on cell-free coagulation and fibrinolysis}

The thrombin generation assay is an accepted evaluation method for anticoagulation by direct oral anticoagulants (DOACs), including the frequently used FXa-I, rivaroxaban. We employed the CAT approach with rivaroxaban-anticoagulated plasma and used CAT-peak to investigate the fluid replacement-induced rivaroxaban dilution. To capture differences in rivaroxaban sensitivity in various types of plasma, we first compared titrations of rivaroxaban in pooled FFP with that in S/D-treated plasma. At 1 pM TF (Fig 1A, left panel), residual CAT-peak in FFP and the more procoagulant S/Dtreated plasma differed clearly. At $200 \mathrm{ng} / \mathrm{mL}$ rivaroxaban e.g., thrombin generation in FFP was negligible, while it was still significant in S/D-treated plasma. Differences between the plasmas were less pronounced at $5 \mathrm{pM}$ TF (Fig 1A, right panel).

We next performed dilution experiments with plasma collected from rivaroxaban-treated patients (a single dose of $10 \mathrm{mg}$ ). The peak 3-h levels of rivaroxaban in patients' 1-6 undiluted plasma were 71 , $98,13,161,81$ and $99 \mathrm{ng} / \mathrm{mL}$, respectively. The response to $1 \mathrm{pM}$ TF of undiluted sample in CAT was below the lowest 2.5 percentile of 40 normal plasmas' for $3 / 6$ patients only, and did not improve upon $50 \%$ dilution with FFP, but for $5 / 6$ patients the CAT response was partially corrected by dilution with S/D-treated plasma (Fig. 1B, left panel). At 5 pM TF (Fig. 1B, right panel) the CAT-peak response of all undiluted patient plasmas, except patient 3 with the lowest rivaroxaban level, were all below the CAT-peak values of pooled FFP and S/D-treated plasma, and the lowest 2.5 percentile of the 40 control normal plasma samples. This finding deviates from the more sensitive CAT response to $1 \mathrm{pM}$ TF. At 5 pM TF, 50\% dilutions of patient plasma with FFP or S/D-treated plasma only marginally improved the CAT-peak.

CAT-peak is a highly sensitive variable both to rivaroxaban and to differences in procoagulant behavior between FFP and S/D-treated plasma, but does not directly reflect fibrin clot formation and 
stability. Influence of fluid replacement on clot formation and stability in rivaroxaban anticoagulated plasma is shown in Figure 2. These experiments were performed at a low TF concentration ( $0.5 \mathrm{pM})$, enabling accurate and sensitive clotting time determination of this non-automated test. To monitor clot stability, we included tPA. Clotting times of all undiluted plasmas derived from rivaroxaban-treated patients, except patient 3 with the lowest rivaroxaban level, were all higher than the values in 15 normal plasma samples, clearly indicating anticoagulation (Fig. 2A, top panels). These prolonged clotting times only marginally improved upon $50 \%$ dilution of rivaroxaban-anticoagulated patient plasma using either pooled FFP or S/D-treated plasma. On the other hand, clot lysis time of rivaroxaban-anticoagulated plasma was in the normal range for most patients, but shortened upon dilution, especially with S/D-treated plasma (Fig. 2A, bottom panels). Influence of dilution at high concentrations of rivaroxaban were examined using FFP spiked with rivaroxaban up to $500 \mathrm{ng} / \mathrm{mL}$ (Fig. 2B). Dilution of rivaroxaban-spiked FFP with either pooled FFP or S/D-treated plasma clearly improved the clotting times (Fig. 2B, top panel), while correction of the clot lysis time, albeit only partial at high rivaroxaban levels, was observed for the pooled FFP, but not for S/D-treated plasma (Fig. 2B, bottom panel).

\section{Hemostatic profile of rivaroxaban-anticoagulated whole blood - comparison between dilution with saline, albumin, FFP and S/D-treaded plasma}

The drawbacks of the CAT assay and the FGCLT are the sample dilution with reagents $(50 \% \mathrm{v} / \mathrm{v}$ in our setup) and the absence of blood cells and platelets. We therefore performed TEG experiments with citrated whole blood where reagents accounted for only $14 \%$ of the total extracellular volume. For logistic reasons, these experiments were performed with whole blood samples of healthy volunteers that were spiked in vitro with rivaroxaban. Plasma compartment of the native and rivaroxabananticoagulated whole blood samples was diluted 2-fold with the resuscitation fluid assuming a hematocrit of $40 \%$ (Fig. 3). Representative TEG tracings are shown in Figure 3A. Dilution of native, citrated whole blood with pooled FFP was without significant effect, whereas dilution with S/D-treated plasma resulted in a marked shortening of the clot lysis time (Fig. 3B). A $50 \%$ dilution of the plasma compartment of native samples with the saline or albumin solution profoundly impaired the TEG tracing, characterized primarily by a reduced maximal amplitude and shortened clot lysis time (Fig. 3). 
Main impact of spiking native citrated whole blood with rivaroxaban was on the reaction time $\mathrm{R}$ (1.8fold prolongation, $\mathrm{p}<0.004$ ). Influence on $\mathrm{R}$-time of the subsequent $50 \%$ dilution (plasma compartment) of rivaroxaban-anticoagulated blood with resuscitation liquid was only marginal, showing some improvement by $\mathrm{NaCl}$ and albumin. Other TEG parameters did not improve. In fact, apart from FFP, all resuscitation fluids led to a further deterioration of the hemostatic profile of rivaroxaban-anticoagulated blood that is mainly due to reducing clot stability.

\section{Role of fibrinolytic inhibitors in fluid replacement under rivaroxaban}

We investigated whether fibrinolysis inhibitors may improve clot stability under rivaroxaban, when using saline or S/D-treated plasma for resuscitation. The antifibrinolytic drug tranexamic acid abrogated clot lysis not only in rivaroxaban-anticoagulated whole blood diluted with saline, but also when diluting with S/D-treated plasma (Table I). Dilution with saline or S/D-treated plasma, decreases, among others, the natural fibrinolysis inhibitor $\alpha_{2}$-antiplasmin. Restoring $\alpha_{2}$-antiplasmin corrected the clot lysis time to correspond minimally diluted whole blood (Table I). Fibrinolytic inhibitors tranexamic acid and $\alpha_{2}$-antiplasmin, as expected, did not affect TEG R-time. On the other hand, both fibrinolytic inhibitors improved maximal amplitude (MA) to some extent, probably due to delayed fibrinolysis (Table I). To negate the effect on clot stability, most resuscitation fluids thus may require additional support of antifibrinolytic drugs.

\section{Procoagulant drug support in fluid replacement under rivaroxaban}

The low maximal amplitude (MA) in our TEG analysis of saline-diluted blood (see Fig. 3) can be interpreted as a shortage of fibrinogen. Therefore, fibrinogen was added to restore its level. This supplementation partially corrected MA but did not influence TEG-R and CLT (Fig 4). We next argued that PCCs may be useful not only to correct for rivaroxaban anticoagulation, but also to negate dilutional coagulopathy. As shown in Figure 4, addition of PCC only had a minor effect on TEG-R, but on the other hand significantly delayed clot lysis. Interestingly, combined use of PCC and FFP resuscitation strongly improved TEG-R, $\alpha$ angle, maximal amplitude and CLT values (Fig 5). This combined resuscitation approach seems more effective at the "trough" rather than at "peak" 
rivaroxaban levels, as indicated by the complete correction of TEG-R at $50 \mathrm{ng} / \mathrm{mL}$ rivaroxaban, whereas the correction was only partial at $300 \mathrm{ng} / \mathrm{mL}$ concentration of rivaroxaban.

The ability of PCC to delay clot lysis was analyzed in more detail using FGCLT. Figure 6 shows an example of the fibrin generation and clot lysis profile of the control and rivaroxaban-spiked normal plasma ( $50 \%$ final volume during measurement). Adding PCC to individual donor FFP ( $n=8)$ spiked with rivaroxaban only modestly corrected the clotting time from $15.1 \pm 0.8 \mathrm{~min}$ to $11.8 \pm 0.4 \mathrm{~min}$. (Table II). Clot stability, on the other hand, increased significantly (1.5-2-fold prolonged the clot lysis time). In our CAT assay this corresponded to a significant improvement of the peak and overcorrection of ETP, while lag time was only minimally affected (Table II). Prolongation of the clot lysis time by PCC was abrogated in the presence of carboxypeptidase inhibitor, suggesting a role for TAFI (Fig. 6). 


\section{DISCUSSION}

In this study, the impact of different resuscitation fluids on hemostatic variables in rivaroxabananticoagulated plasma was explored, using both in vitro spiked samples and samples from hip arthroplasty patients taking rivaroxaban. Although rivaroxaban is diluted when transfusing resuscitation fluids, the anticoagulant effect is not nullified. We observed only a partial, rivaroxaban concentration dependent correction of the clotting time in vitro when applying FFP or S/D-treated plasma in a 1:1 ratio to endogenous, rivaroxaban-anticoagulated plasma. Furthermore, resuscitation fluids also dilute endogenous plasma components. This ultimately will result in a dilutional coagulopathy when using crystalloid fluids, further deteriorating coagulation that is already hampered by rivaroxaban. Ongoing rivaroxaban anticoagulation thus complicates the management of patients who experience acute traumatic bleeds, suggesting the need for additional measures including the use of FXa-I-neutralizing drugs or hemostatic agents that bypass the anticoagulant.

Hemostasis is not only determined by thrombin generation and subsequent fibrin clot formation but also by processes involved in clot dissolution. In both the absence and presence of rivaroxaban, we observed increased clot lysis when diluting with S/D-treated plasma. Clot lysis was further augmented when diluting with albumin or saline. It is known that manufacturing of S/D-treated plasma reduces the natural fibrinolysis inhibitor $\alpha_{2}$-antiplasmin levels to $40 \%$, but concomitant enhanced clot lysis can be corrected by replacing $\alpha_{2}$-antiplasmin. ${ }^{10,26}$ Similarly, when we restored $\alpha_{2}$-antiplasmin or abrogated fibrinolysis by tranexamic acid, clot lysis in saline-diluted plasma was either normalized or strongly inhibited, respectively. To negate the disrupting effect on clot stability, most resuscitation fluids therefore may require additional but temporary support by antifibrinolytic drugs, as has been shown beneficial also clinically early in association with severe trauma. ${ }^{27}$

$S / D$-treated plasma is not only low in $\alpha_{2}$-antiplasmin but also has gained procoagulant properties during manufacturing that mainly emerge at low TF. ${ }^{10,13}$ Subsequently, residual thrombin generation in CAT at low TF in rivaroxaban-anticoagulated S/D-treated plasma was higher compared with FFP. 
Fluid replacement with S/D-treated plasma in case of rivaroxaban anticoagulation thus may be preferred over FFP as both the rivaroxaban dilution and the addition of procoagulant plasma enhances hemostasis. Under our experimental conditions however, the FGCLT-clotting time in rivaroxabananticoagulated plasma diluted with FFP or S/D-treated plasma did not differ. It should be noted that in these plasma-based methods, CAT and FGCLT, the required assay reagents and buffer further dilute the samples, resembling saline-induced dilutional coagulopathy. In TEG, a much smaller dilution factor is used ( $14 \%$ in our setup). However, also in TEG at low TF, clot formation in rivaroxabananticoagulated plasma diluted with FFP or S/D-treated plasma did not differ. Whether indeed rivaroxaban-treated patients benefit from S/D-treated plasma as a resuscitation fluid over FFP remains to be investigated.

Blood dilution diminished fibrinogen concentration, as well as impaired clot strength and structure, which are associated with traumatic coagulopathy. The poorest survival is evident among individuals having both decreased functional fibrinogen concentration and clot strength. ${ }^{28,29}$ Due to its diminished effects on thrombin formation, rivaroxaban anticoagulation compatibly associates with lower fibrin network density, i.e. by formation of thick, loosely woven strands, instead of more resilient thin, tightly packed fibrin strands. ${ }^{30-34}$ We found that fibrinogen addition indeed improves the TEG variable MA in agreement with previous studies, suggesting fibrinogen as a limiting factor. ${ }^{35,36}$ The beneficial effect of fibrinogen added to saline-diluted whole blood anticoagulated with rivaroxaban, however, was marginal or absent for the TEG variables of R-time and CLT. Additional PCC support improved CLT, but not MA and R-time. In contrast, PCC used in conjunction with FFP can correct for rivaroxaban anticoagulation, as indicated by improved R-time and MA. Yet, correction to normal levels was only achieved at low rivaroxaban level of $50 \mathrm{ng} / \mathrm{mL}$, corresponding to typical trough concentrations. In a clinical cohort study, most patients experiencing major bleeding under rivaroxaban anticoagulation benefited from PCC. ${ }^{37}$ Our data indicate that clinically induced dilutional coagulopathy by saline during rivaroxaban use cannot be fully corrected by currently available pro-hemostatic agents, illustrating the need of a specific rivaroxaban reversal agent. 
The use of anticoagulation predisposes patients to a significant risk of surgery or trauma-associated bleed. The most urgent treatment in life-threatening bleed is to secure intravascular volume. Unfortunately, the use of crystalloid resuscitation fluids easily leads to dilutional coagulopathy, impairing both coagulation and fibrinolysis. In our study we showed accelerated fibrinolysis by rivaroxaban. Hypocoagulability and hyperfibrinolysis associate with multiorgan failure and mortality. ${ }^{38,39} \mathrm{~A}$ characteristic diamond pattern in TEG with rapid clot formation and subsequent fibrinolysis, is the harbinger of death in patients with $100 \%$ mortality. ${ }^{40}$ Countering volume loss with FFP combined with PCC resuscitation is probably most beneficial, as the increased thrombin generation produces stronger fibrin network and enhances TAFI activation, thereby inhibiting fibrinolysis. When S/D plasma is used, we recommend supplementation with tranexamic acid to correct fibrinolysis.

Major limitation is the in vitro nature of our study. Trauma-induced coagulopathy is not solely driven by the dilutional effect of fluid resuscitation. Severe tissue damage triggers a cascade of events leading to impaired platelet activity and endothelial dysfunction..$^{2,4,41}$ In our study, these effects of endothelium or platelets were not assessed. In addition, in traumatic coagulopathy the TF component is enhanced. ${ }^{2}$ In our in vitro experiments we used TF concentrations ranging from $0.5 \mathrm{pM}$ to $5 \mathrm{pM}$. It remains unclear whether these concentrations mimic local in vivo exposure to TF due to injury. Hyperfibrinolysis was anticipated by tPA addition. On the other hand, the plasma samples of our study originated from patients after orthopedic surgery, which may harbor some specific trauma features, including the proinflammatory postoperative state and mimicking of bone fractures. Yet, patients had taken only the first dose of rivaroxaban at the time of sampling (steady state was not reached). Nevertheless, the orthopedic patients were clinically stable at time of sampling.

In conclusion, we do not recommend saline or albumin solution as the rescue therapy to achieve hemostasis in individuals on rivaroxaban anticoagulation. Our in vitro results suggest that the best resuscitation benefit while on rivaroxaban may be achieved with PCC in combination with FFP. S/D plasma use alone or in combination with PCC albeit enhancing thrombin generation, may predispose patients to increased fibrinolysis due to its diminished $\alpha_{2}$-antiplasmin concentration. Early 
supplementation with tranexamic, as recommended in general during major trauma, may benefit in these instances.

\section{Addendum}

$R L$ and $H J M B$ were responsible for the initial concept of the study. TH, MM, RL and HJMB contributed to the design of the study. TH and MM were responsible for collecting patient samples $\mathrm{TH}, \mathrm{MZ}$ and HJMB performed experiments, acquired and analyzed data. TH, JCMM, RL and HJMB interpreted the data and wrote the paper. All authors read and approved the final manuscript. 


\section{References}

(1) Mahambrey T, Pendry K, Nee A, et al. Critical care in emergency department: massive haemorrhage in trauma. Emerg Med J 2013;30:9-14.

(2) Cap A, Hunt BJ. The pathogenesis of traumatic coagulopathy. Anaesthesia 2015;70 Suppl 1:96101, e32-4.

(3) Davenport RA, Brohi K. Cause of trauma-induced coagulopathy. Curr Opin Anaesthesiol 2016;29:212-219.

(4) Chang R, Cardenas JC, Wade CE, et al. Advances in the understanding of trauma-induced coagulopathy. Blood 2016;128:1043-1049.

(5) Myburgh JA, Mythen MG. Resuscitation fluids. N Engl J Med 2013;369:1243-1251.

(6) Long B, Koyfman A. Red Blood Cell Transfusion in the Emergency Department. J Emerg Med 2016;51:120-130.

(7) McCormack PL. Tranexamic acid: a review of its use in the treatment of hyperfibrinolysis. Drugs 2012;72:585-617.

(8) Johansson PI, Stensballe J, Oliveri R, et al. How I treat patients with massive hemorrhage. Blood 2014;124:3052-3058.

(9) Hellstern P, Solheim BG. The Use of Solvent/Detergent Treatment in Pathogen Reduction of Plasma. Transfus Med Hemother 2011;38:65-70.

(10) Pitkänen H, Jouppila A, Mowinckel MC, et al. Enhanced thrombin generation and reduced intact protein S in processed solvent detergent plasma. Thromb Res 2015;135:167-174.

(11) Porta CR, Nelson D, McVay D, et al. The effects of tranexamic acid and prothrombin complex concentrate on the coagulopathy of trauma: an in vitro analysis of the impact of severe acidosis. $J$ Trauma Acute Care Surg 2013;75:954-960.

(12) Joseph B, Aziz H, Pandit V, et al. Prothrombin complex concentrate versus fresh-frozen plasma for reversal of coagulopathy of trauma: is there a difference? World J Surg 2014;38:1875-1881.

(13) van Beers JJ, van Egmond LT, Wetzels RJ, et al. Increased coagulation and fibrinolytic potential of solvent-detergent plasma: a comparative study between Omniplasma and fresh frozen plasma. Vox Sang 2016;111:33-42.

(14) Eriksson BI, Borris LC, Dahl OE, et al. A once-daily, oral, direct Factor Xa inhibitor, rivaroxaban (BAY 59-7939), for thromboprophylaxis after total hip replacement. Circulation 2006;114:2374-2381.

(15) Eriksson BI, Borris LC, Friedman RJ, et al. Rivaroxaban versus enoxaparin for thromboprophylaxis after hip arthroplasty. N Engl J Med 2008;358:2765-2775.

(16) Patel MR, Mahaffey KW, Garg J, et al. Rivaroxaban versus warfarin in nonvalvular atrial fibrillation. N Engl J Med 2011;365:883-891.

(17) Burnett AE, Mahan CE, Vazquez SR, et al. Guidance for the practical management of the direct oral anticoagulants (DOACs) in VTE treatment. J Thromb Thrombolysis 2016;41:206-232.

(18) Siegal DM, Curnutte JT, Connolly SJ, et al. Andexanet Alfa for the Reversal of Factor Xa Inhibitor Activity. N Engl J Med 2015;373:2413-2424.

(19) Eerenberg ES, Kamphuisen PW, Sijpkens MK, et al. Reversal of rivaroxaban and dabigatran by prothrombin complex concentrate: a randomized, placebo-controlled, crossover study in healthy subjects. Circulation 2011;124:1573-1579. 
(20) Levi M, Moore KT, Castillejos CF, et al. Comparison of three-factor and four-factor prothrombin complex concentrates regarding reversal of the anticoagulant effects of rivaroxaban in healthy volunteers. J Thromb Haemost 2014;12:1428-1436.

(21) Perzborn E, Gruber A, Tinel H, et al. Reversal of rivaroxaban anticoagulation by haemostatic agents in rats and primates. Thromb Haemost 2013;110:162-172.

(22) Zhou W, Zorn M, Nawroth P, et al. Hemostatic therapy in experimental intracerebral hemorrhage associated with rivaroxaban. Stroke 2013;44:771-778.

(23) Herzog E, Kaspereit F, Krege W, et al. Correlation of coagulation markers and 4F-PCC-mediated reversal of rivaroxaban in a rabbit model of acute bleeding. Thromb Res 2015;135:554-560.

(24) Bakhtiari K, Kamphuisen PW, Mancuso ME, et al. Clot lysis phenotype and response to recombinant factor VIla in plasma of haemophilia A inhibitor patients. Br J Haematol 2013;162:827835.

(25) Dinkelaar J, Molenaar PJ, Ninivaggi M, et al. In vitro assessment, using thrombin generation, of the applicability of prothrombin complex concentrate as an antidote for Rivaroxaban. J Thromb Haemost 2013;11:1111-1118.

(26) Saadah NH, van der Meer PF, Brinkman HJM, et al. Effect of solvent/detergent-treated pooled plasma on fibrinolysis in reconstituted whole blood. Transfusion 2017.

(27) CRASH-2 trial collaborators, Shakur H, Roberts I, et al. Effects of tranexamic acid on death, vascular occlusive events, and blood transfusion in trauma patients with significant haemorrhage (CRASH-2): a randomised, placebo-controlled trial. Lancet 2010;376:23-32.

(28) Shen L, Lorand L. Contribution of fibrin stabilization to clot strength. Supplementation of factor XIII-deficient plasma with the purified zymogen. J Clin Invest 1983;71:1336-1341.

(29) Wolberg AS, Campbell RA. Thrombin generation, fibrin clot formation and hemostasis. Transfus Apher Sci 2008;38:15-23.

(30) Wolberg AS. Thrombin generation and fibrin clot structure. Blood Rev 2007;21:131-142.

(31) Undas A, Ariens RA. Fibrin clot structure and function: a role in the pathophysiology of arterial and venous thromboembolic diseases. Arterioscler Thromb Vasc Biol 2011;31:e88-99.

(32) Incampo F, Carrieri C, Galasso R, et al. Effect of warfarin treatment on thrombin activatable fibrinolysis inhibitor (TAFI) activation and TAFI-mediated inhibition of fibrinolysis. J Thromb Haemost 2013;11:315-324.

(33) Varin R, Mirshahi S, Mirshahi P, et al. Whole blood clots are more resistant to lysis than plasma clots--greater efficacy of rivaroxaban. Thromb Res 2013;131:e100-9.

(34) Bembenek JP, Niewada M, Siudut J, et al. Fibrin clot characteristics in acute ischaemic stroke patients treated with thrombolysis: the impact on clinical outcome. Thromb Haemost 2017;117:14401447.

(35) Schafer N, Driessen A, Bauerfeind U, et al. In vitro effects of different sources of fibrinogen supplementation on clot initiation and stability in a model of dilutional coagulopathy. Transfus Med 2016;26:373-380.

(36) Carr ME. Fibrin formed in plasma is composed of fibers more massive than those formed from purified fibrinogen. Thromb Haemost 1988;59:535-539.

(37) Majeed A, Ågren A, Holmström M, et al. Management of rivaroxaban- or apixaban-associated major bleeding with prothrombin complex concentrates: a cohort study. Blood 2017;130:1706-1712.

(38) Muller MC, Balvers K, Binnekade JM, et al. Thromboelastometry and organ failure in trauma patients: a prospective cohort study. Crit Care 2014;18:687-014-0687-6.

(39) Branco BC, Inaba K, Ives C, et al. Thromboelastogram evaluation of the impact of hypercoagulability in trauma patients. Shock 2014;41:200-207. 
(40) Chapman MP, Moore EE, Moore HB, et al. The "Death Diamond": Rapid thrombelastography identifies lethal hyperfibrinolysis. J Trauma Acute Care Surg 2015;79:925-929.

(41) Kutcher ME, Redick BJ, McCreery RC, et al. Characterization of platelet dysfunction after trauma. J Trauma Acute Care Surg 2012;73:13-19. 


\section{FIGURES}

A

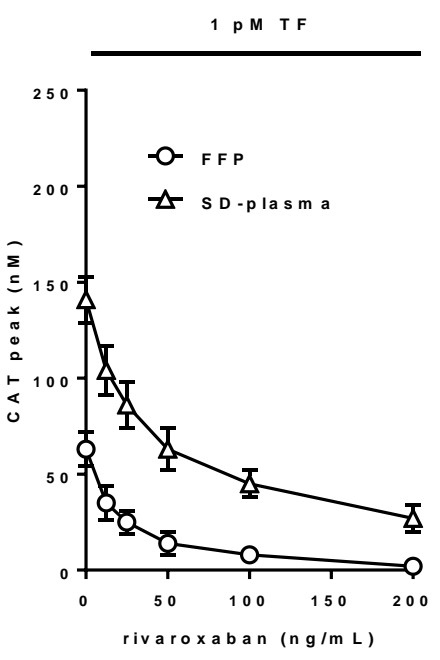

B

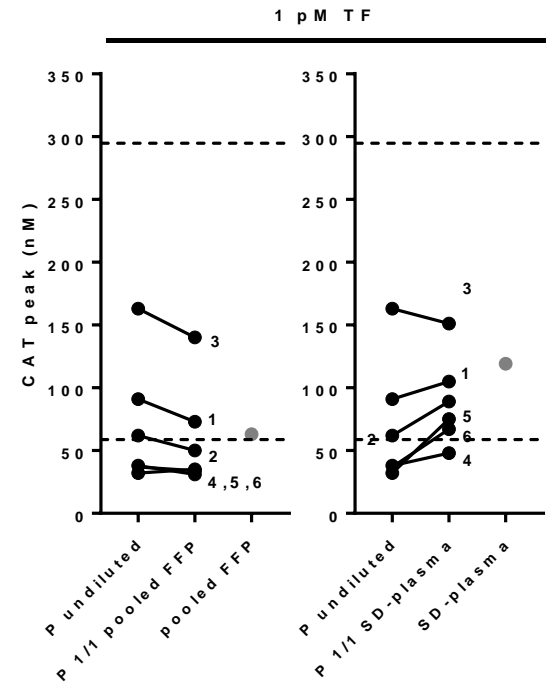

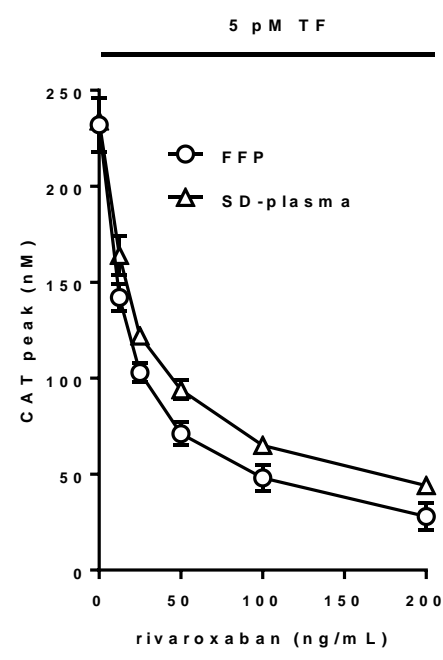

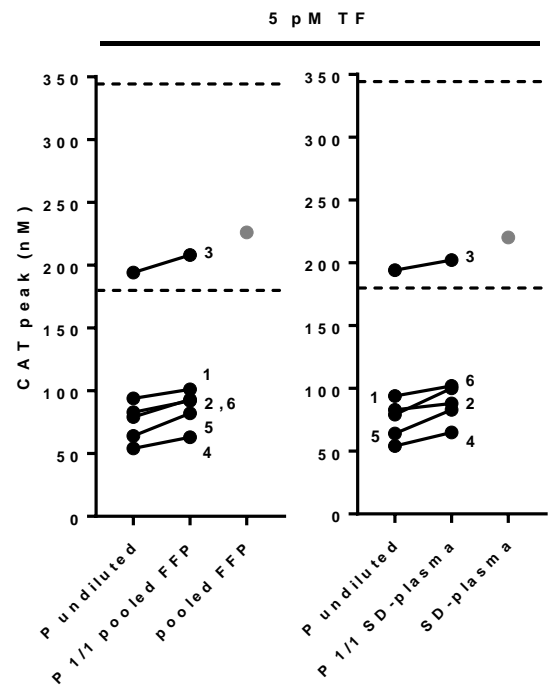

Figure 1. Influence of plasma replacement-induced reduction of rivaroxaban concentration on

thrombin generation. (A) Rivaroxaban was titrated in pooled FFP and S/D-treated plasma. Samples were analyzed by CAT in the presence of $1 \mathrm{pM}$ TF and $5 \mathrm{pM}$ TF. Average data $( \pm \mathrm{SD})$ of three experiments are shown. (B) Citrated plasma samples from 6 rivaroxaban-anticoagulated patients $(P)$. Levels of rivaroxaban in native patient plasma (1-6) were: 71 (1), 98 (2), 13 (3), 161 (4), 81 (5) and 99 (6) $\mathrm{ng} / \mathrm{respectively.} \mathrm{One} \mathrm{aliquot} \mathrm{was} \mathrm{left} \mathrm{undiluted,} \mathrm{while} \mathrm{the} \mathrm{other} \mathrm{two} \mathrm{were} \mathrm{diluted} 50 \%$ with either pooled FFP or S/D-treated plasma. Samples were analyzed as described above. Black bullet: patient plasma before and after $50 \%$ dilution. Grey bullet: diluent (either pooled FFP or S/D-treated plasma). Dotted line: 2.5 th-97.5th percentile interval for 40 normal plasma samples. 
A
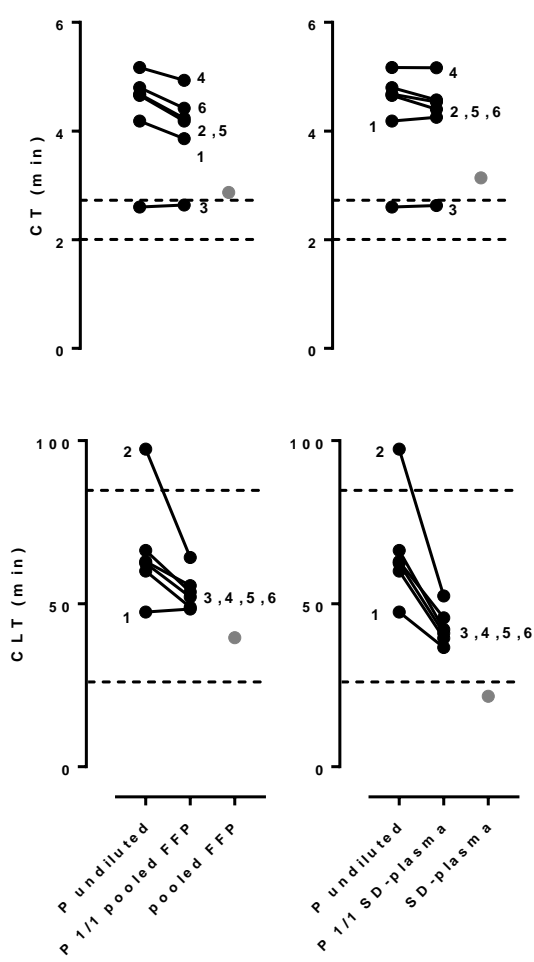

B
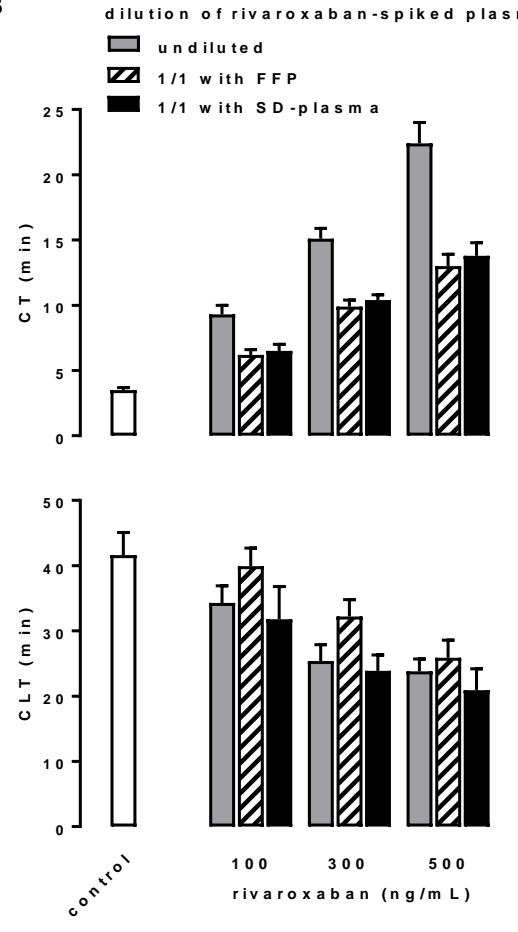

Figure 2. Consequences of plasma replacement-induced reduction of rivaroxaban concentration on fibrin clot formation and fibrinolysis. (A) Citrated plasma samples from 6 rivaroxaban-anticoagulated patients (P 1-6). Levels of rivaroxaban in native patient plasma were 71 (1), 98 (2), 13 (3), 161 (4), 81 (5) and 99 (6) ng/respectively. One aliquot was left undiluted, while two others were diluted $50 \%$ with either pooled FFP or S/D-treated plasma. Samples were analyzed by the fibrin generation clot lysis test (FGCLT) and the variables fibrin generation clotting time (CT) and clot lysis time (CLT) were derived. Black bullet: patient plasma before and after 50\% dilution. Grey bullet: diluent (either pooled FFP or S/D-treated plasma). Dotted line: $2.5^{\text {th }}-97.5^{\text {th }}$ percentile interval for 15 normal plasma samples. Please note that the CT of S/D-treated plasma is somewhat increased compared to normal, illustrating increased procoagulant properties of this manufactured plasma 10,13 . Please also note that CLT of S/D-treated plasma is below the normal control range due to reduced $\alpha 2-$ antiplasmin ${ }^{10,26}$. (B) Pooled FFP (white bar) was spiked with 100, 300 and $500 \mathrm{ng} / \mathrm{mL}$ rivaroxaban. One aliquot was left undiluted (grey bar), while two others were diluted $50 \%$ with either pooled FFP (hatched bar) or S/D-treated plasma (black bar). Mean \pm SD of three separate experiments. 


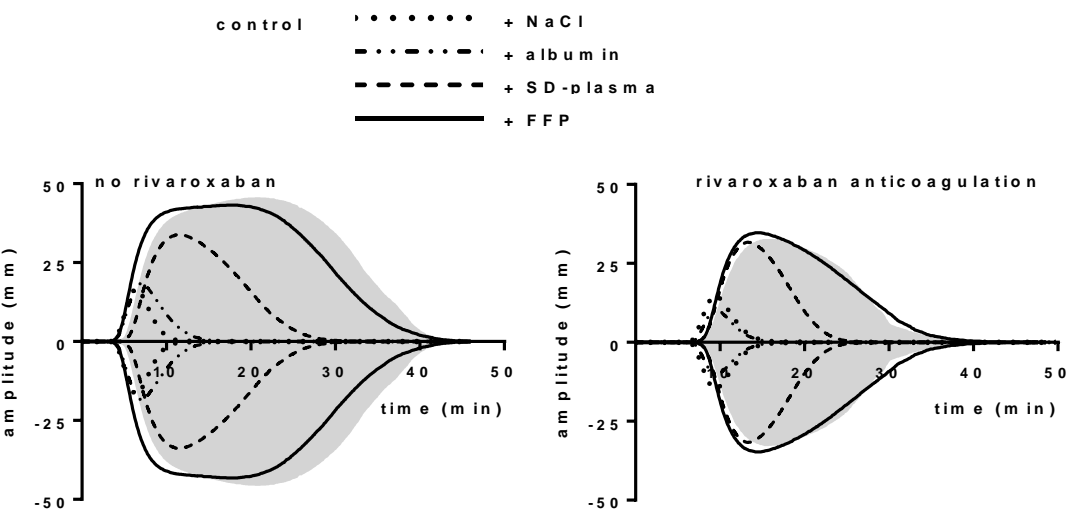

B

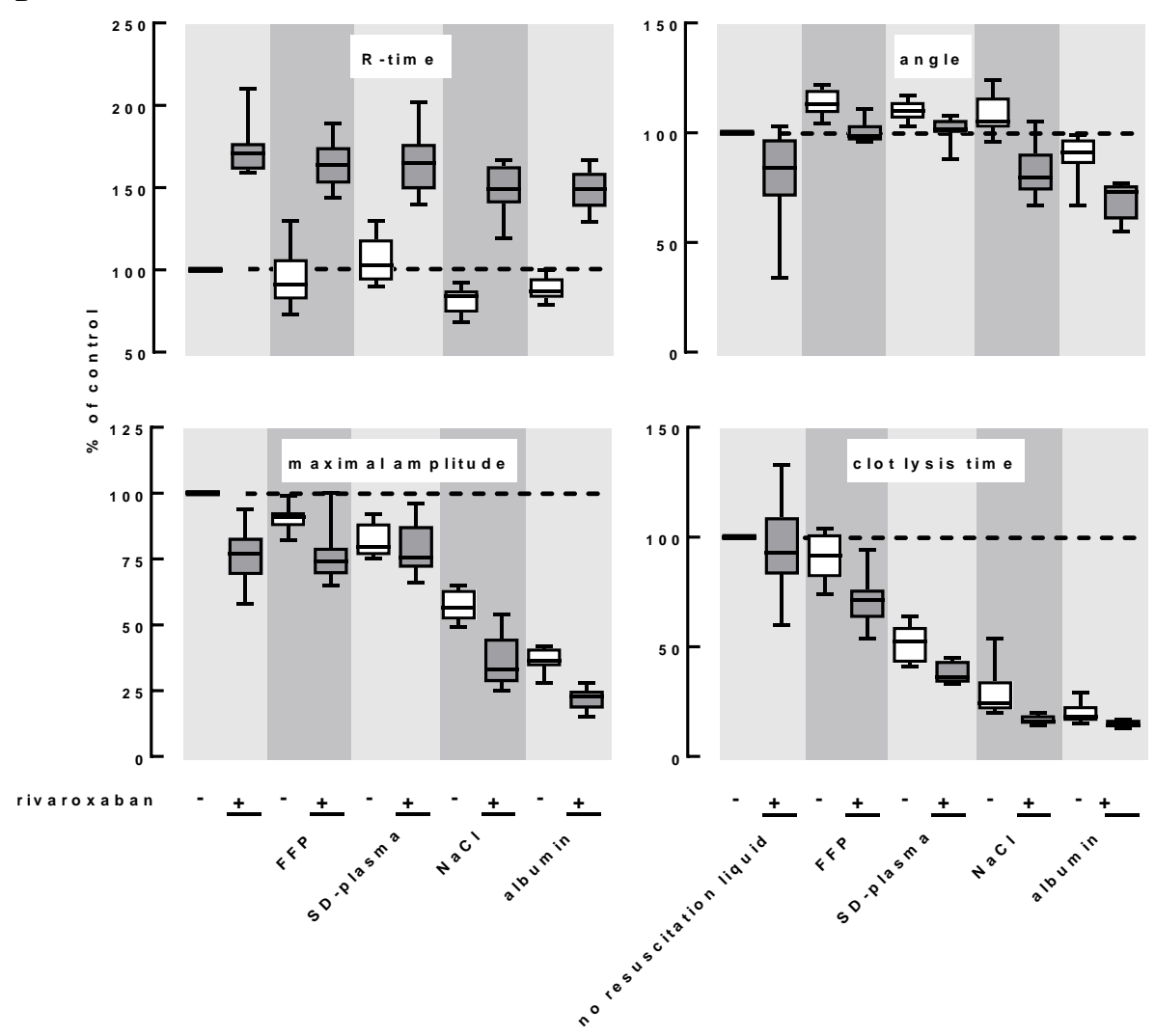

Figure 3. Influence of high-dose rivaroxaban on the effect of resuscitation fluids on

thromboelastography (TEG) tracings in the presence of tPA. Individual donor citrated whole blood was spiked with rivaroxaban to a final concentration of $300 \mathrm{ng} / \mathrm{mL}$ in donor plasma $(0.3 \% \mathrm{v} / \mathrm{v} \mathrm{DMSO})$ or its DMSO solvent, assuming a hematocrit of $40 \%$. Spiked samples were aliquoted. One aliquot was examined undiluted in TEG, while the other aliquots were examined after the addition of one of the following resuscitation fluids: none, $0.9 \% \mathrm{NaCl}, 4 \%$ albumin, S/D-treated plasma, pooled FFP. Volume of resuscitation fluid added was equal to the volume of donor plasma in the whole blood sample. TEG 
was performed in the presence of $1 \mathrm{pM}$ tissue factor (TF) and $200 \mathrm{ng} / \mathrm{mL}$ tissue plasminogen activator (tPA). Concentrations of these reagents are based on the final extracellular volume during

measurement. A: Representative TEG tracings. B: Plots showing $25^{\text {th }}-75^{\text {th }}$ percentile boxes with min to max whiskers (white box, no rivaroxaban spiking; grey box, rivaroxaban spiked sample) of TEG variables obtained from 9 individual whole blood samples. TEG variables are represented as $\%$ of variables obtained with the corresponding native sample without rivaroxaban and undiluted with resuscitation fluid. 
A

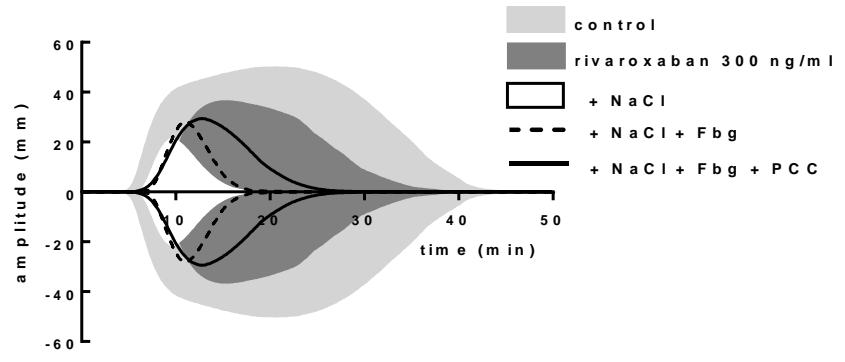

B
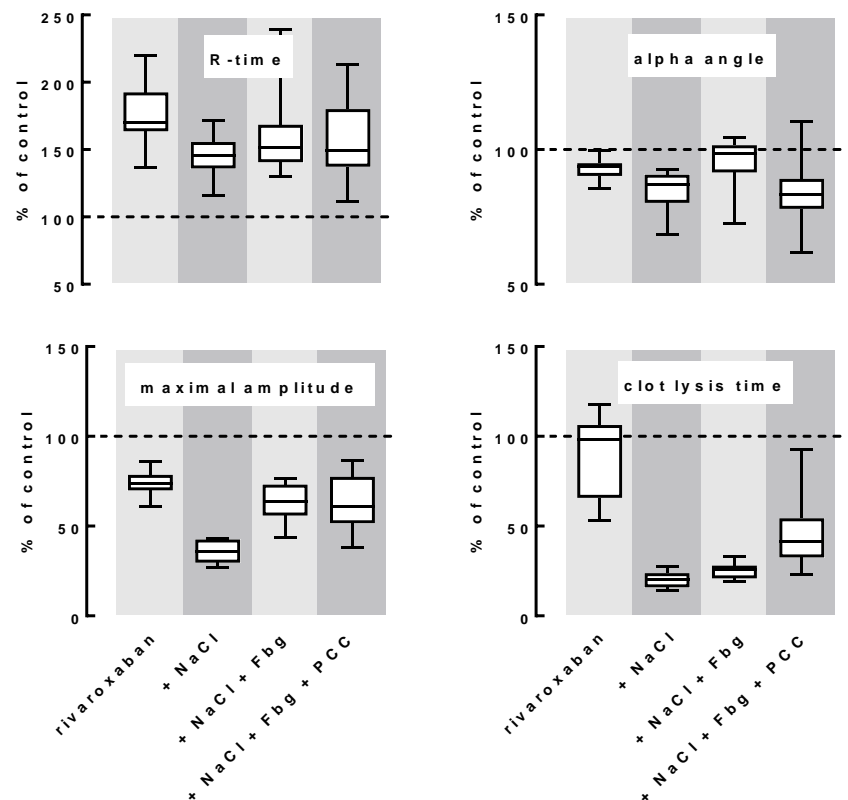

Figure 4. Effect of fibrinogen and PCC support on thromboelastography (TEG) during dilutional coagulopathy with high-dose rivaroxaban spiking. Individual donor citrated whole blood was spiked with rivaroxaban (or its solvent DMSO) to a final rivaroxaban concentration in donor plasma of $300 \mathrm{ng} / \mathrm{mL}$ ( $0.3 \% \mathrm{v} / \mathrm{v} \mathrm{DMSO})$, assuming a hematocrit of $40 \%$. Spiked samples were aliquoted. Three aliquots were diluted with $0.9 \% \mathrm{NaCl}$ and two of them supplemented with either fibrinogen (Fbg) alone or together with PCC. Volume of resuscitation fluid added was equal to the volume of donor plasma in the whole blood sample. Fbg was added to the saline compartment to a concentration of $2 \mathrm{mg} / \mathrm{ml}$. PCC was added to equal a concentration in plasma of $2 \mathrm{IU} / \mathrm{mL}$. TEG was performed in the presence of $1 \mathrm{pM}$ tissue factor (TF) and $200 \mathrm{ng} / \mathrm{mL}$ tissue plasminogen activator (TPA). A: Representative TEG tracings. B: Plots showing $25^{\text {th }}-75^{\text {th }}$ percentile boxes with min to max whiskers of TEG variables obtained from whole blood samples from 11 different donors. TEG variables are represented as $\%$ of variables obtained with the corresponding sample without rivaroxaban spiking and undiluted with a resuscitation fluid. 
A
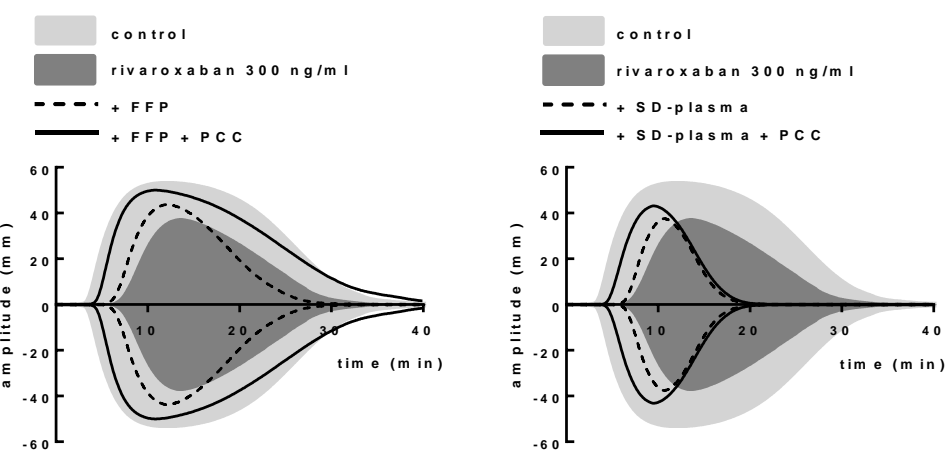

B
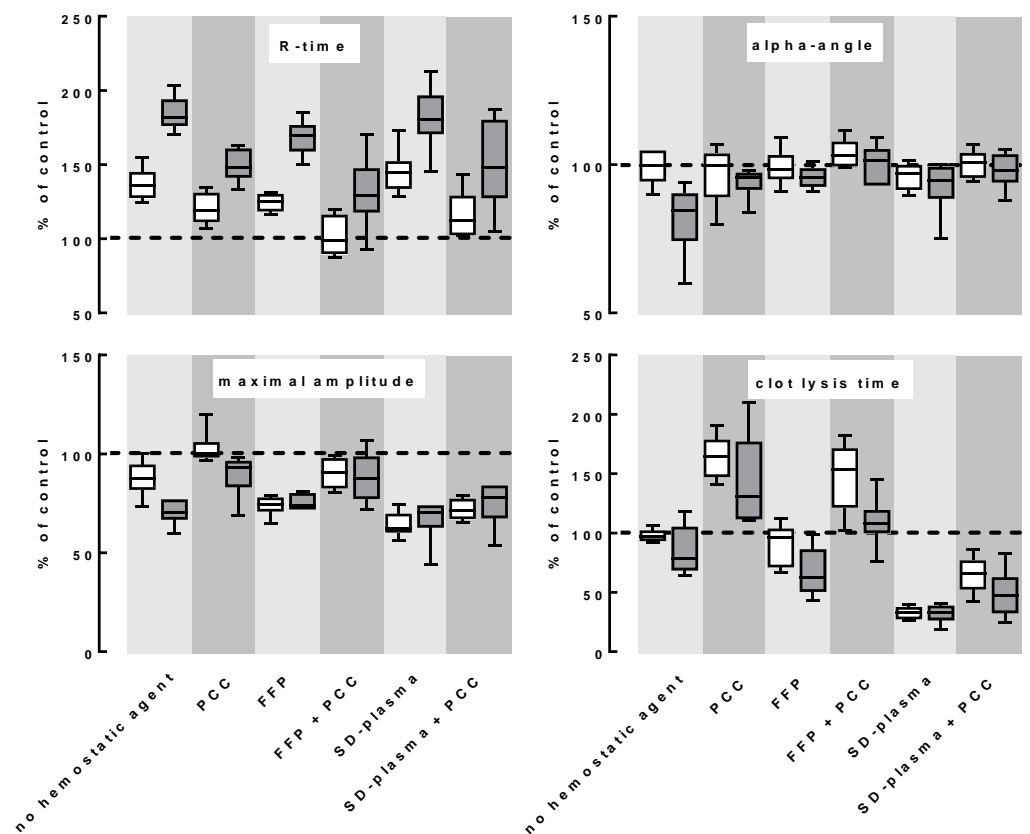

Figure 5. Additive effect of plasma and prothrombin complex concentrate (PCC) on the thromboelastography (TEG) readout of rivaroxaban-spiked (low- and high-dose) whole blood. Individual donor citrated whole blood from 6 volunteers was spiked with rivaroxaban to a final concentration of $50 \mathrm{ng} / \mathrm{mL}$ (white boxes). Similarly, whole blood samples from 6 other volunteers were spiked with $300 \mathrm{ng} / \mathrm{mL}$ rivaroxaban (grey boxes). Added hemostatic agents were PCC (2 IU/mL donor plasma), pooled FFP or S/D-treated plasma, and a combination of plasma and PCC. The volume of added pooled plasma was equal to the volume of individual donor plasma. TEG was performed in the presence of $1 \mathrm{pM}$ tissue factor (TF) and $200 \mathrm{ng} / \mathrm{mL}$ tissue plasminogen activator (tPA). A:

Representative TEG tracings. B: Plots show $25^{\text {th }}-75^{\text {th }}$ percentile boxes with min to max whiskers. 

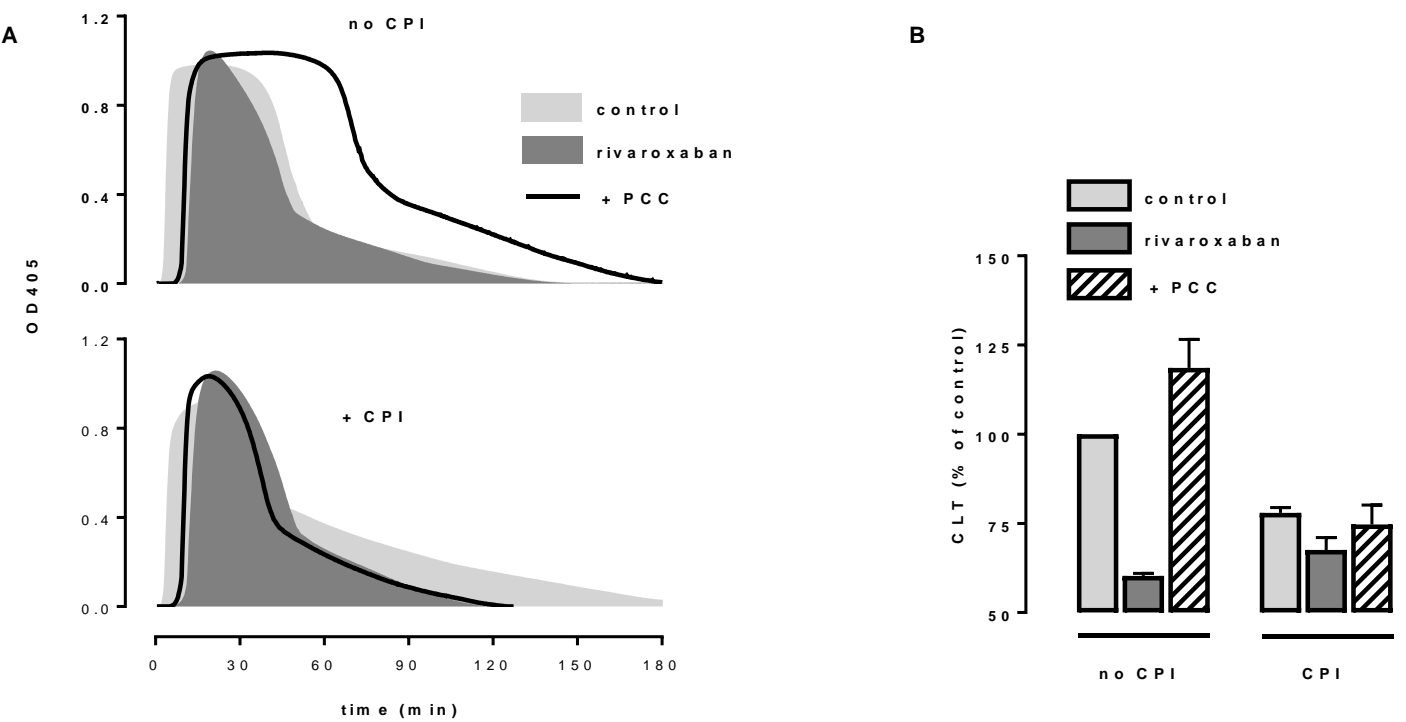

Figure 6. Fibrin formation and dissolution in high-dose rivaroxaban-anticoagulated plasma and the effect of PCC. Pooled normal plasma and pooled normal plasma spiked with rivaroxaban (300 $\mathrm{ng} / \mathrm{mL}$ ) was analyzed by the fibrin generation clot lysis time test in the absence and presence of PCC (A, top panel). PCC was added to equal a concentration in plasma of $2 \mathrm{IU} / \mathrm{mL}$. In parallel, samples were analyzed in the presence of $30 \mu \mathrm{g} / \mathrm{mL}$ carboypeptidase inhibitor (CPI) (A, bottom panel). Figure B shows the mean \pm SD CLT of three independently performed experiments. 
TABLES AND LEGENDS

Table l. Correction of dilutional coagulopathy in thromboelastography (TEG) using inhibitors of fibrinolysis

\begin{tabular}{lccc}
\hline & R-time (\%) & MA (\%) & CLT (\%) \\
\hline TXA support: & & & \\
& & & \\
rivaroxaban & $196(145-220)$ & $77(62-104)$ & $85(62-137)$ \\
rivaroxaban + saline & $155(111-170)$ & $40(20-57)$ & $19(17-43)$ \\
rivaroxaban + saline + TXA & $153(111-212)^{\mathrm{ns}}$ & $113(91-141) \dagger$ & $291(88-495) \dagger$ \\
& & & \\
rivaroxaban & $200(169-212)$ & $76(60-104)$ & $83(70-137)$ \\
rivaroxaban + S/D plasma & $180(159-219)$ & $67(57-112)$ & $33(27-75)$ \\
rivaroxaban + S/D plasma + TXA & $178(168-219)^{\mathrm{ns}}$ & $111(119-175)^{*}$ & $283(215-440)^{*}$
\end{tabular}

$\underline{\alpha 2-a n t i p l a s m i n ~ r e s t o r e: ~}$

$\begin{array}{llll}\text { rivaroxaban } & 189(155-238) & 70(62-84) & 85(77-106) \\ \text { rivaroxaban + saline } & 143(128-205) & 38(24-54) & 20(12-26) \\ \text { rivaroxaban + saline + A2AP } & 156(130-200)^{\mathrm{ns}} & 48(35-68) \dagger & 93(61-129) \dagger \\ & & & \\ \text { rivaroxaban } & 196(169-238) & 71(62-84) & 84(70-106) \\ \text { rivaroxaban + S/D plasma } & 188(168-223) & 67(54-74) & 33(21-41) \\ \text { rivaroxaban + S/D plasma + A2AP } & 200(169-233)^{\mathrm{ns}} & 78(68-94) \dagger & 70(48-82) \dagger\end{array}$

Individual donor citrated whole blood was spiked with solvent (DMSO) or rivaroxaban to a final concentration of $300 \mathrm{ng} / \mathrm{mL}(0.3 \% \mathrm{v} / \mathrm{v}$ DMSO), assuming a hematocrit of $40 \%$. Spiked samples were aliquoted. One undiluted aliquot was examined in TEG, while the other aliquots were examined after 
adding saline or solvent/detergent (S/D)-treated plasma (volume added was equal to the volume of donor plasma in the whole blood sample) and tranexamic acid (TXA, $2.5 \mu \mathrm{g} / \mathrm{mL}$ ) or $\alpha 2$-antiplasmin (A2AP: $1 \mathrm{IU} / \mathrm{mL}$ in saline compartment, $0.6 \mathrm{IU} / \mathrm{mL}$ in S/D-treated plasma compartment). TEG variables are represented as \% of variables obtained with the corresponding native, control sample not spiked with rivaroxaban. CLT did not show normal distribution (Shapiro-Wilk normality test). Data are expressed as median (range) and were obtained from 7-10 different donors. ${ }^{*} p<0.05, \uparrow p<0.005$, ns not significant (Wilcoxon matched pairs signed rank test). 
Table II. Effect of PCC on coagulation and fibrinolysis in plasma spiked with rivaroxaban

\begin{tabular}{lccccc}
\hline & $50 \mathrm{ng} / \mathrm{ml}$ rivaroxaban & & \multicolumn{2}{c}{$300 \mathrm{ng} / \mathrm{ml}$ rivaroxaban } \\
\cline { 2 - 3 } \cline { 6 - 6 } & - & PCC & & - & PCC \\
\hline Thrombin generation & & & & \\
\hline CAT - lag time (\%) & $198 \pm 26$ & $171 \pm 18^{*}$ & & $278 \pm 50$ & $254 \pm 25^{\mathrm{ns}}$ \\
CAT - peak (\%) & $36 \pm 7$ & $115 \pm 14 \dagger$ & & $17 \pm 6$ & $52 \pm 7 \dagger$ \\
CAT - ETP (\%) & $91 \pm 4$ & $206 \pm 29 \dagger$ & & $73 \pm 14$ & $189 \pm 19 \dagger$
\end{tabular}

Fibrin clot formation and dissolution

\begin{tabular}{lllrr} 
CT (\%) & $174 \pm 20$ & $187 \pm 18^{\text {ns }}$ & $316 \pm 38$ & $325 \pm 30^{\text {ns }}$ \\
CLT (\%) & $101 \pm 9$ & $152 \pm 11 \dagger$ & $82 \pm 12$ & $149 \pm 10 \dagger$ \\
\hline
\end{tabular}

Individual donor citrated plasma $(\mathrm{n}=8)$ was aliquoted and spiked with solvent (DMSO) or rivaroxaban to a final concentration of 50 and $300 \mathrm{ng} / \mathrm{mL}(0.3 \% \mathrm{v} / \mathrm{v}$ DMSO). Thrombin generation assay (CAT) and fibrin generation and clot lysis test (FGCLT) were performed in the absence or presence of PCC (2 $\mathrm{IU} / \mathrm{mL}$ plasma) at a final plasma concentration of $50 \% \mathrm{v} / \mathrm{v}$. Data expressed as $\%$ of control (without rivaroxaban and PCC) show a normal distribution (D'Agostino \& Pearson normality test), and are presented as mean $\pm S D$. ${ }^{*} p<0.05, \dagger P<0.0005$, ns not significant (t-test).PCC $=$ prothrombin complex concentrate, $\mathrm{CAT}=$ calibrated automated thrombogram, $\mathrm{CT}=$ clotting time, $\mathrm{CLT}=$ clot lysis time . 